\title{
Growth and resorption of bubbles in magma
}

E.W. LlEWELLIN ${ }^{1}$, J.P.COUMANS ${ }^{1}$, F.B. WADSWORTH ${ }^{1}$, M.C.S. HUMPHREYS ${ }^{1}$, K.J. DOBSON ${ }^{2}$, A. AllabAR ${ }^{3}$, R.J. BROOKER $^{4}$, J.E. GARDNER ${ }^{5}$, T. CONNOLLEY ${ }^{6}$

${ }^{1}$ Department of Earth Sciences, Durham University, Durham, UK (email: ed.llewellin@durham.ac.uk)

${ }^{2}$ Civil and Environmental Engineering, University of Strathclyde, Glasgow, UK

${ }^{3}$ Department of Geosciences, University of Tuebingen, Germany

${ }^{4}$ School of Earth Sciences, University of Bristol, Bristol, UK

${ }^{5}$ Jackson School of Geosciences, University of Texas at Austin, USA

${ }^{6}$ Diamond Light Source Ltd., Harwell Science and Innovation Campus, Didcot, UK

The rate and timing of bubble growth in magma is an important control on eruption style, determining whether or not magma fragments to produce an explosive eruption. Bubbles nucleate, grow, shrink, and de-nucleate in magma in response to changes in pressure and temperature, and these changes may be recorded in the vesicle textures, and in the spatial distribution and speciation of water 'frozen into' the glass in eruption products. We have developed a numerical model for growth and resorption of bubbles in magma, and validated it against experiments across a wide range of conditions. The model allows for arbitrary temperature and pressure pathways, and accounts for the impact of spatial variations in water content on diffusivity and viscosity.

Textures in natural, vesicular volcanic rocks are often used to interpret eruptive processes. Similarly, high-pressure, high-temperature experiments are used to probe bubble growth processes, via textural and chemical analysis of the experimental products. However, in both cases, the analysed samples have cooled from magmatic temperatures before analysis, providing a window for thermally-driven bubble shrinkage and resorption to modify the sample. Consequently, interpretations of syn-eruptive and syn-experimental processes must account for changes during cooling.

We present results from in situ experiments under synchrotron-source tomography, which demonstrate the thermally driven growth and resorption of bubbles in magma at one atmosphere. The model is applied to $4 \mathrm{D}$ textural data, and used to investigate the role of bubble-bubble interactions in modifying growth and resorption behaviour. We also apply the model to re-analyse the results of high-temperature, highpressure experiments, and demonstrate the importance of accounting for thermal resorption during cooling. 